\title{
Validation of a mouse xenograft model system for gene expression analysis of human acute lymphoblastic leukaemia
}

\author{
Amy L Samuels 1,2, Violet K Peeva1', Rachael A Papa33, Marin J Firth2,4, Richard W Francis, Alex H Beesley1,2, \\ Richard B Lock ${ }^{3}$ and Ursula R Kees*1,2
}

\begin{abstract}
Background: Pre-clinical models that effectively recapitulate human disease are critical for expanding our knowledge of cancer biology and drug resistance mechanisms. For haematological malignancies, the non-obese diabetic/severe combined immunodeficient (NOD/SCID) mouse is one of the most successful models to study paediatric acute lymphoblastic leukaemia (ALL). However, for this model to be effective for studying engraftment and therapy responses at the whole genome level, careful molecular characterisation is essential.

Results: Here, we sought to validate species-specific gene expression profiling in the high engraftment continuous ALL NOD/SCID xenograft. Using the human Affymetrix whole transcript platform we analysed transcriptional profiles from engrafted tissues without prior cell separation of mouse cells and found it to return highly reproducible profiles in xenografts from individual mice. The model was further tested with experimental mixtures of human and mouse cells, demonstrating that the presence of mouse cells does not significantly skew expression profiles when xenografts contain $90 \%$ or more human cells. In addition, we present a novel in silico and experimental masking approach to identify probes and transcript clusters susceptible to cross-species hybridisation.

Conclusions: We demonstrate species-specific transcriptional profiles can be obtained from xenografts when high levels of engraftment are achieved or with the application of transcript cluster masks. Importantly, this masking approach can be applied and adapted to other xenograft models where human tissue infiltration is lower. This model provides a powerful platform for identifying genes and pathways associated with ALL disease progression and response to therapy in vivo.
\end{abstract}

\section{Background}

Understanding the complex molecular pathways leading to disease is critical for the development of effective treatment regimes and novel drug targets. Due to research and resource limitations associated with the use of primary patient material, pre-clinical models are essential to expand our knowledge of cancer biology and for the evaluation of new drugs. For pre-clinical testing, cell lines cultured in vitro have been extensively used but their ability to recapitulate primary disease is limited. Therefore, more relevant disease models are of critical importance.

\footnotetext{
*Correspondence: ursula@ichr.uwa.edu.au

${ }^{1}$ Division of Children's Leukaemia and Cancer Research, Telethon Institute for Child Health Research, Perth, Western Australia

Full list of author information is available at the end of the article
}

An ideal model would mimic the proliferation and dissemination of cancer cells that occur in vivo and behave in a similar manner in response to chemotherapeutic drug treatment. The non-obese diabetic/severe combined immunodeficient (NOD/SCID) xenograft mouse model is currently one of the most successful models with which to study haematological malignancies such as acute lymphoblastic leukaemia (ALL) [1], whereby patient bone marrow leukaemia cells are directly transplanted into recipient NOD/SCID mice [2]. The kinetics of engraftment reflects the human disease, leading to bone marrow (BM) infiltration, followed by migration to the spleen, peripheral blood and other haematopoietic organs [2-4]. 
For ALL, although cure rates are exceeding $75 \%$, the development of drug resistance is poorly understood and remains a major cause of morbidity and mortality in children [5]. Importantly, much of our knowledge of the mechanisms underlying drug resistance has been generated in vitro using immortalised cancer cell lines. The extent to which cell lines retain features of the original disease in vivo is a matter of debate [6]. Thus relevant in vivo, pre-clinical models that recapitulate human disease are critical to delineate resistance mechanisms and improve survival.

Primary leukaemia cells engrafted into NOD/SCID mice appear to retain many of the phenotypic and genotypic features of the original specimen [2,7-10]. Moreover, their drug resistance profile to conventional chemotherapeutics mirrors that of the patient clinical response $[2,10]$. Importantly, comparisons have shown that such xenografts more closely resemble their tumour type of origin than in vitro cell lines and have been accurate in predicting efficacious drug combinations and clinically active therapeutics [11-14].

Continuous xenografts can be established by transplanting cells harvested from the spleen of engrafted animals into secondary and tertiary recipient mice [10]. Utilising continuous ALL NOD/SCID xenografts the effects of chemotherapy drugs can be assessed at the molecular level. Thus, the aim of the current study was to characterise gene expression profiling in the continuous ALL xenograft so that it can be used as a model for the development of therapy resistance in vivo. We have previously demonstrated the clinical relevance of gene-expression profiling through the successful identification of markers predictive of ALL disease outcome, drug-resistance and relapse in a number of primary ALL patient cohorts [15-21]. However, to validate the xenograft model system for transcriptional analysis three critical issues needed to be addressed. Firstly, we needed to determine the most appropriate engrafted xenograft tissue for analysis. BM is more commonly isolated from patients, however, the spleen in xenograft mice contains at least sevenfold more leukaemia cells, which makes isolation of these cells more practical for analysis. Thus, we were interested in establishing whether the same gene expression profiles can be obtained from engrafted spleen and BM. Secondly, we wanted to address the reproducibility of the engraftment in both the BM and spleen of independent mice. Phenotypic evidence suggests engraftment in the continuous mouse model is reproducible [10], however, this has not been examined at the transcriptional level. Finally, when testing the expression of human xenografts we wanted to measure the effect of the host murine tissue. Studies from other xenograft models have demonstrated cross-species hybridisation of mouse RNA to human specific microarrays. Although not extensively characterised, such studies suggest the potential skewing of human gene expression profiles [22-26]. The previous studies were performed using Affymetrix expression arrays designed to target the 3' end of the gene. This region shows the most divergence between mouse and human. To date, the extent of cross hybridisation using the Affymetrix whole transcript Human Gene 1.0 ST array has not been assessed. The goal of the present study was therefore to validate the use of gene expression profiling in the ALL NOD/SCID xenograft model so that it can be used as a pre-clinical model of relapse.

\section{Results \\ Comparison of BM/spleen transcriptional profiles of ALL xenografts}

Our previous studies have demonstrated that there are no morphological differences in ALL cells engrafted to the $\mathrm{BM}$ or spleen of NOD/SCID mouse xenografts [2]. We therefore conducted a microarray investigation to determine if the transcriptional profiles for engrafted spleen and $\mathrm{BM}$ are also analogous. The previously described continuous ALL-16 xenograft established in NOD/SCID mice was used to obtain engrafted $B M$ and spleen tissue [2]. At harvest, high levels of human CD45+ cell engraftment were reached with $>99 \%$ human cells infiltrating the mouse spleen and BM. Using Affymetrix GeneChip Human Gene 1.0 ST ${ }^{\circ}$ arrays the transcriptional profile of engrafted BM tissue was compared to spleen tissue following normalisation. We compared BM and spleen from four engrafted mice. Pearson correlation coefficients were calculated and MvA plots, which display the log difference between BM and spleen expression levels on the $\mathrm{y}$-axis to the average signal intensity on the $\mathrm{x}$-axis, were generated. The MvA plot demonstrates little variation in expression levels between the BM and spleen arrays, only one transcript cluster showed a two fold difference in expression (Figure 1A). The Pearson correlation coefficients calculated between $\mathrm{BM}$ and spleen further show high concordance, ranging between 0.99234-0.99683 for the four xenografts (Table 1). Consistent with these results unsupervised hierarchical clustering revealed that the xenografts do not cluster according to $\mathrm{BM}$ or spleen tissue origin (Figure 1B). To determine differential expression between engrafted BM and spleen a moderated $t$-test was performed. To account for the small sample size a linear model with empirical Bayes estimation (limma) and Benjamini-Hochberg correction was applied $[27,28]$. With this approach only three of the 28,869 genes profiled, ATP binding cassette A (ABCA1), ATP binding cassette $G(A B C G 1)$ and insulin-like growth factor binding protein 5 (IGFBP5) were identified as significantly differentially expressed $(\mathrm{p}<0.05)$. Together these results demonstrate a high level of transcriptional concordance 
Table 1: Pearson correlation summary statistics showing the engraftment reproducibility between bone marrow and spleen xenografts from four independent mice.

\begin{tabular}{cccc}
\hline & ALL. BM - ALL.BM & ALL.SP -ALL.SP & ALL. BM - ALL.SP \\
\hline Range & 0.99394 & 0.99445 & 0.99234 \\
n (comparisons) & 0.99657 & 0.99576 & 0.99683 \\
SD & 6 & 6 & 16 \\
Mean & 0.00102 & 0.00052 & 0.00120 \\
SE & 0.99556 & 0.99529 & 0.99496 \\
\hline
\end{tabular}

$\mathrm{ALL}=$ Acute lymphoblastic leukaemia, $\mathrm{BM}=$ bone marrow, $\mathrm{SP}=$ spleen, $\mathrm{SE}=$ standard error.

between engrafted BM and spleen suggesting both could be used interchangeably for gene expression analysis.

\section{Reproducibility of engraftment}

To establish the reproducibility of engraftment in the continuous ALL model, we compared the gene expression profiles of ALL-16 xenografts from the spleen and $\mathrm{BM}$ of four independent mice. Pearson correlation coefficients were calculated to compare the reproducibility of the mean signal intensity between biological replicates (Additional file 1). All transcript clusters were compared for pairwise combinations of microarrays (Table 1) and correlations between tissues showed little variation. These results demonstrate excellent reproducibility in the transcriptional profiles of independent mice transplanted with the ALL-16 xenograft. Importantly, this high level of reproducibility was observed when comparing BM or spleen xenografts among independent mice. This high concordance suggests engraftment of leukaemia cells in $\mathrm{BM}$ and spleen is highly reproducible in the continuous ALL xenograft model.

\section{Analysis of cross species hybridisation in the ALL xenograft model}

Recovery of human tissue from the engrafted murine host introduces the possibility of contamination from the host mouse cells. The ALL xenograft mouse represents a high engraftment model. At harvest, BM, spleen and peripheral blood all reach high levels of human CD45+ cells, with BM and spleen typically containing > 97\% (with a range from 90-99\%) [2]. Therefore, BM/spleen xenograft preparations are estimated to contain 1-10\% mouse cells. We developed an experimental approach to evaluate the effect of residual mouse cells in the presence of $90-100 \%$ human ALL cells using the whole transcript Affymetrix microarray platform.

Graded cell mixtures were prepared with 100\% engrafted human leukaemia cells (ALL.100) and spleen cells prepared from non-engrafted mice (NES) to derive 95\% human (ALL.95) and 90\% human (ALL.90) cell mix- tures. All three mixtures were analysed by microarray in triplicate to detect transcriptional changes due to mouse RNA. When comparing ALL.100, with ALL.95 or ALL.90 highly concordant gene expression profiles were obtained with little variation shown by MvA plots of the mean signal intensity (Figure 2A). Moreover, Pearson correlation coefficients comparing ALL.100 with ALL.95 or ALL.90 showed no difference between the cell mixtures (Table 2). Principal components analysis (PCA) demonstrated that there is no clustering between any of the samples including the three biological replicates for each cell mixture, further showing the high degree of similarity between all samples examined (Figure 2B). To compare differential gene expression a moderated t-test (limma) with Benjamini-Hochberg correction was performed. This approach did not identify any significant $(\mathrm{p}<0.05)$ differential gene expression between ALL.100 and ALL.95 or ALL.90. These results indicate that the presence of $<10 \%$ mouse cells does not significantly skew human gene expression signatures. Importantly, other xenograft models show different levels of mouse cells in engrafted tissue and therefore may require a masking approach to control crossspecies hybridisation. We tested this using two complementary masking approaches.

\section{Masking cross species hybridisation}

We first tested the hybridisation of pure mouse nonengrafted spleen (NES) to human arrays (Figure 3). We found that the overall raw signal intensity and signal to noise ratio (SNR) of mouse transcripts was significantly reduced compared to the hybridisation of the human samples (signal intensity 115.36-329.47 and SNR 0.7-1.67 respectively). The signal distribution of the hybridising targets showed a reduced frequency for high intensity values for mouse NES compared to ALL.100 (Figure 3). A small percentage of transcript clusters, however, resulted in very high signals for the mouse NES samples, these were selected as candidates for the masking approach (Figure 4). Taking the top $1 \%$ of normalised mean signal expression we identified 312 transcript clusters with a 
A

MvA plot of BM versus Spleen

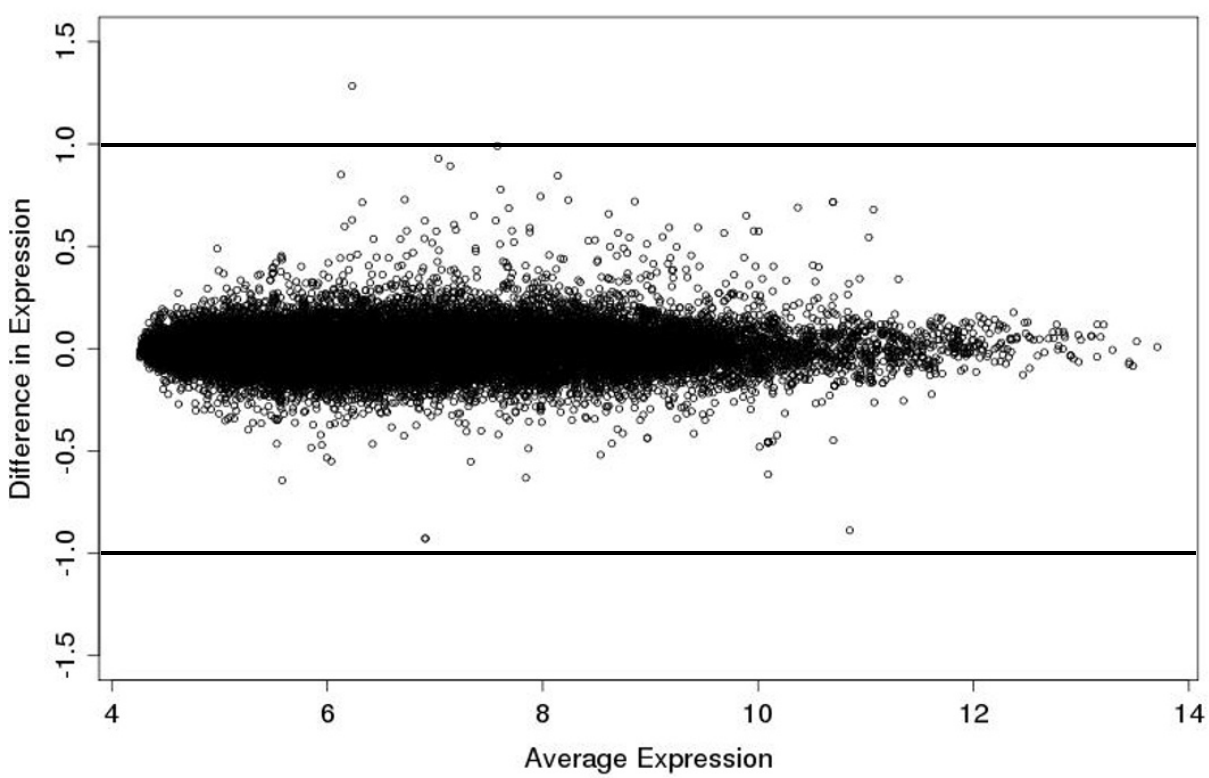

B

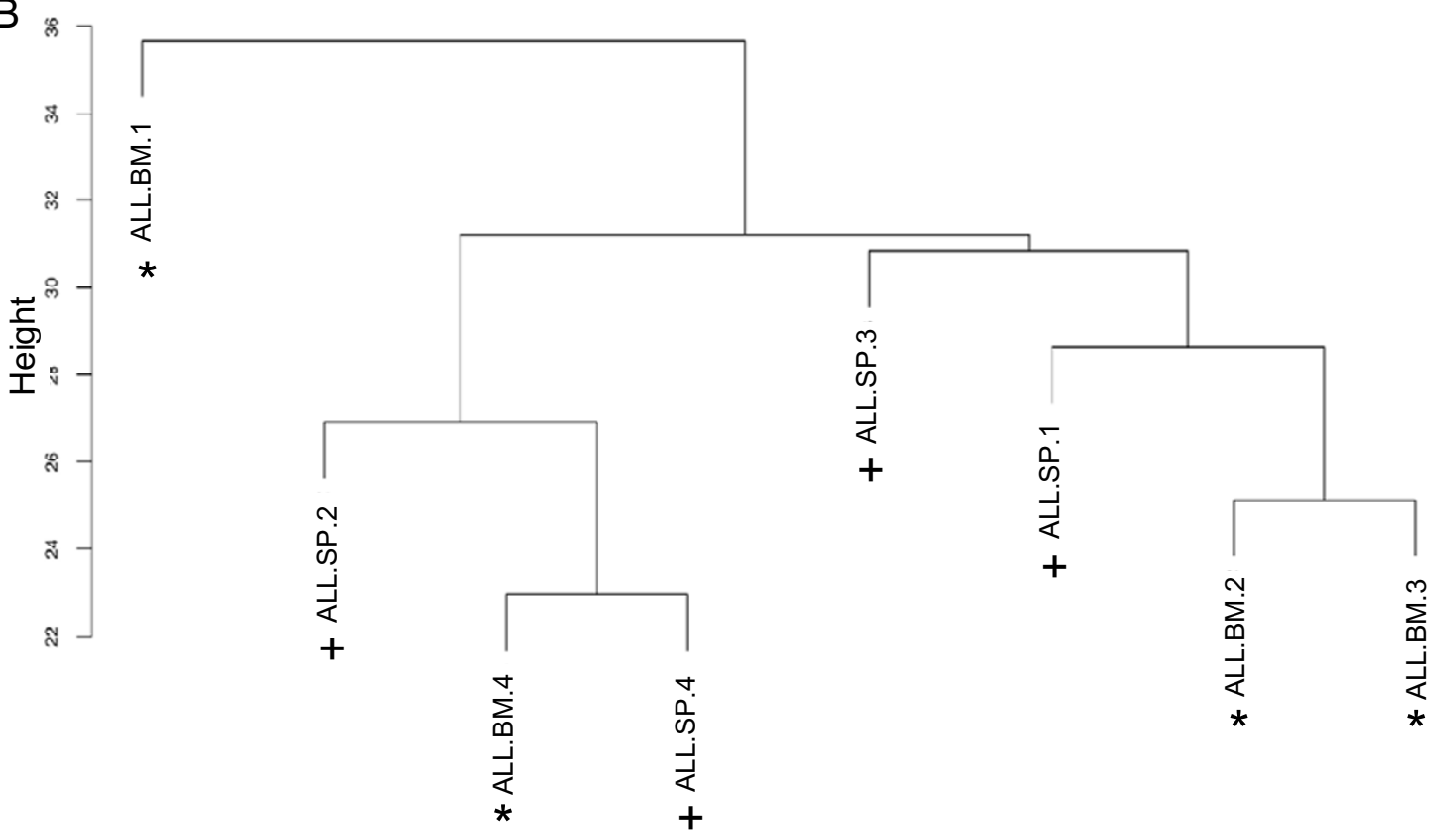

Figure 1 Comparison of differential expression levels between engrafted ALL-16 bone marrow $(n=4)$ and spleen $(n=4)$ xenografts. A MvA plot comparing expression differences with expression averages for all transcript clusters. Plots were generated using RMA normalised log 2 signal intensities, lines represent 2-fold difference $\left(\log _{2}=1\right)$. B Unsupervised hierarchical clustering of engrafted ALL-16 bone marrow $\left(^{*}\right)$ and spleen $(+)$ xenografts from four NOD/SCID mice. 
Table 2: Pearson correlation summary statistics showing the reproducibility of mixing experiments between ALL.90 ( $90 \%$ human $A L L)(n=3)$ versus ALL.100 (100\% human $A L L)(n=8)$ and ALL.95 (95\% human ALL) $(n=3)$ versus ALL.100 from independent mice.

\begin{tabular}{ccc}
\hline & ALL.90 - ALL.100 & ALL.95 - ALL. 100 \\
\hline Range & 0.99316 & 0.99247 \\
$\mathrm{n}$ (comparisons) & 0.99731 & 0.99712 \\
Mean & 24 & 24 \\
$\mathrm{SE}$ & 0.99537 & 0.99527 \\
\hline
\end{tabular}

$\mathrm{ALL}=$ Acute lymphoblastic leukaemia, $\mathrm{SE}=$ standard error.

signal intensity higher than 7.56 (log base 2 scale) (Figure 4B) (Additional file 2).

We then identified transcript clusters theoretically susceptible to cross-species hybridisation due to their associated probes having high identity to mouse transcripts. We identified 75,300 probes, which matched the mouse genome with $100 \%$ identity and/or with a single base mismatch. Of the 28,869 human transcript clusters targeted by the Human Gene ST 1.0 array our filtering method revealed 1,085 ( 3.75\%) transcript clusters as candidates susceptible to cross-species hybridisation (Additional file 2).

Of the 312 experimentally derived transcript clusters, 117 (37.5\%) were also identified using the in silico approach, highlighting a degree of overlap between the two approaches. Both the experimental and in silico masking approaches were combined resulting in 1280 (4.4\%) transcript clusters to be masked from the total gene expression signals (Additional file 2). The combined masking approach was applied to ALL.90 and compared to the ranked signal intensity of ALL.100 (Figure 5). Following application of the combined mask, calculation of the mean squared distance (MSD) between ALL.100 and ALL.90 revealed a decrease in variability (MSD =

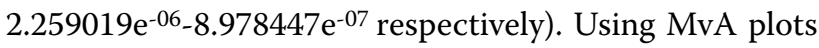
we applied each of the in silico, experimental and combined masking approaches to the ALL.100 versus ALL.90 comparison (Figure 6). Application of the combined approach removed several of the outliers and thus variability enabling us to reduce cross-species hybridisation and improve concordance between the ALL.100 and ALL.90 transcriptional profiles.

\section{Discussion}

The current study sought to address the sensitivity of gene expression profiling for human xenografts growing in mice without prior cell separation. Mouse cells, present in xenografts can be separated using lengthy depletion protocols and fluorescent activated cell sorting (FACS) which can have deleterious affects on transcrip- tional profiles. Several studies, using a variety of xenograft mouse models, have previously examined crossspecies hybridisation without prior cell separation [2226]. These studies were performed using Affymetrix expression arrays designed to target the 3 ' end of genes. The 3 ' expression arrays are thought to be relatively insensitive to cross-species hybridisation as there is greater than 10\% DNA divergence between mouse and human within the 3' untranslated region (UTR). In contrast, the Human gene 1.0 ST array queries the whole transcript, giving a more accurate reflection of gene expression. Using the whole transcript platform, our results show that mouse cells do not significantly skew cancer expression profiles when xenografts contain 90\% or more human cells. Although mouse RNA was found to cross-hybridise to the whole transcript arrays in the absence of human target, when tested in the presence of human RNA (> 90\%), the effect on the transcriptional profiles was minimal. Our microarray analysis and theoretical modelling indicated that the design of the whole transcript Affymetrix chips is effective enough to limit aberrant cross-hybridising mouse RNA. Therefore, without prior cell separation, profiling xenografts on whole transcript arrays is possible for high engraftment haematological models.

We developed experimental and in silico derived gene sets to mask probes and transcript clusters potentially susceptible to cross-species hybridisation. Application of the transcript cluster masks to the gene expression profiles of the human/mouse graded mixtures, improved results by removing outliers and reducing variability. While the derived masking approaches outlined in this study were used for a high engraftment model, this masking approach could be applied and adapted to other xenograft model systems where human tissue infiltration is below $90 \%$.

The kinetics of engraftment in the NOD/SCID ALL xenograft model reflect the human disease with BM infiltration, followed by migration to the spleen, peripheral blood and other haematopoietic organs [2-4]. Impor- 


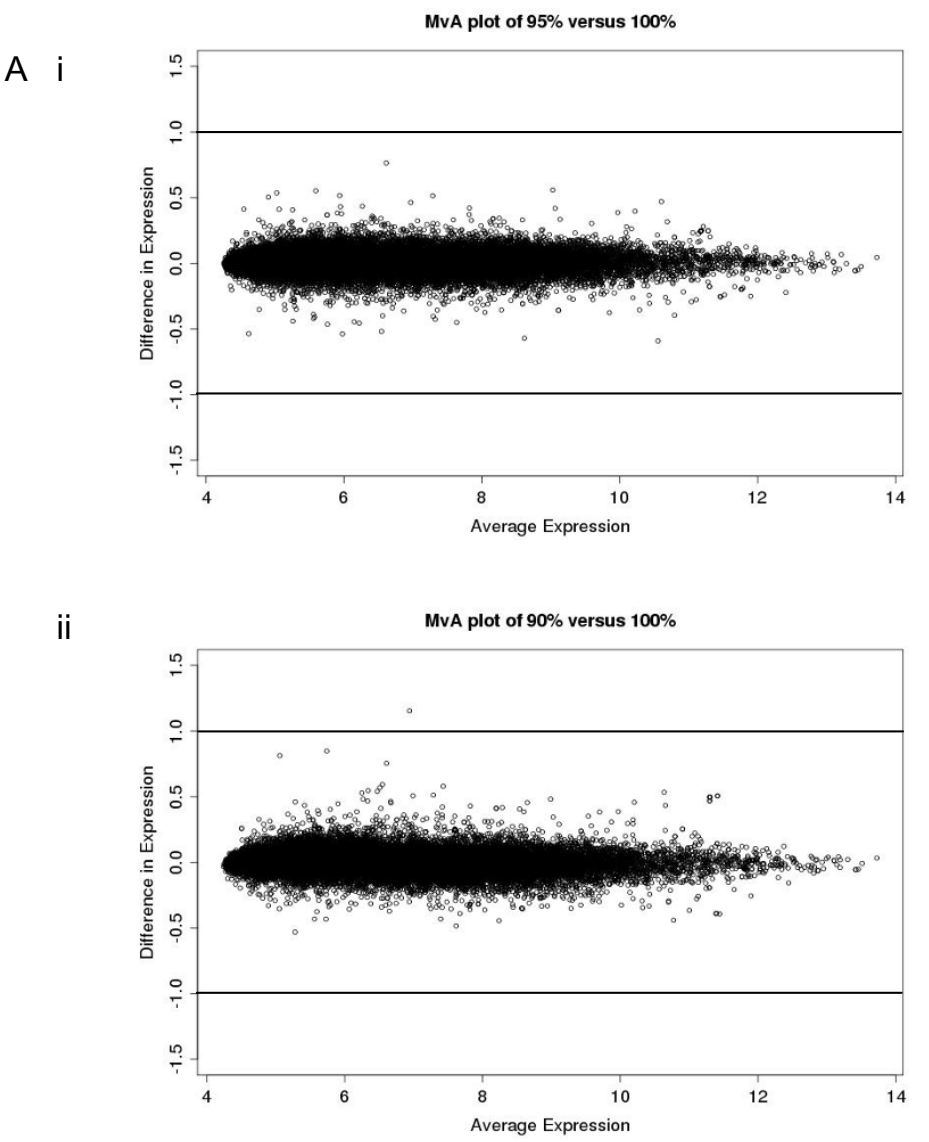

B

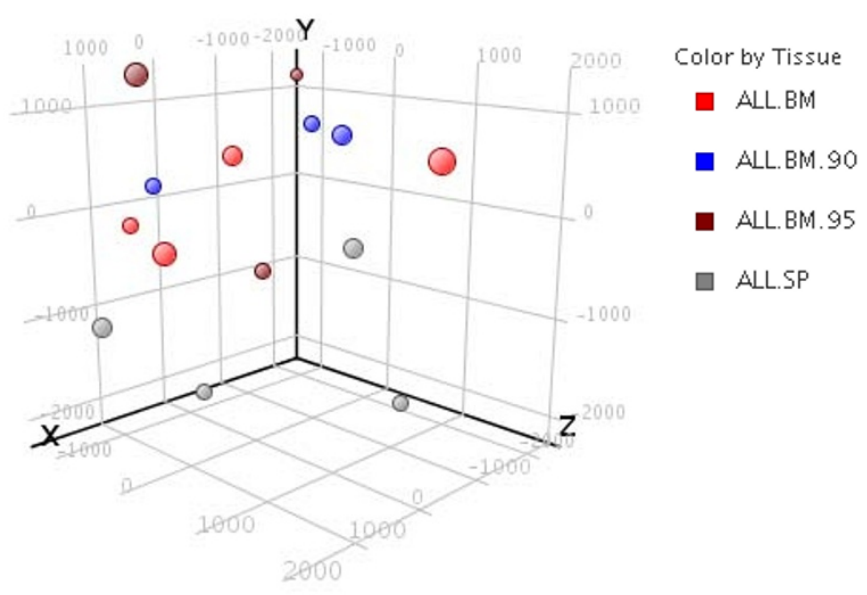

Figure 2 Assessment of differential expression after mixing ALL-16 xenografts with mouse cells (NES). A MvA plots comparing i ALL.100 (100\% human ALL) $(n=8)$ with ALL.95 (95\% human ALL) $(n=3)$ and ii ALL.100 with ALL.90 ( $90 \%$ human) $(n=6)$, MvA plot comparing expression differences with expression averages for all transcript clusters. Plots were generated using RMA normalised $\log _{2}$ signal intensities, lines represent $2-$ fold difference $\left(\log _{2}=1\right)$. B Principle component analysis of global gene-expression profiles from ALL engrafted bone marrow, spleen tissues and cell mixtures of ALL.95 (95\% human) and ALL.90 (90\% human). Principle components were extracted using all transcript clusters. Four principle components were calculated, of which the first three are shown. 


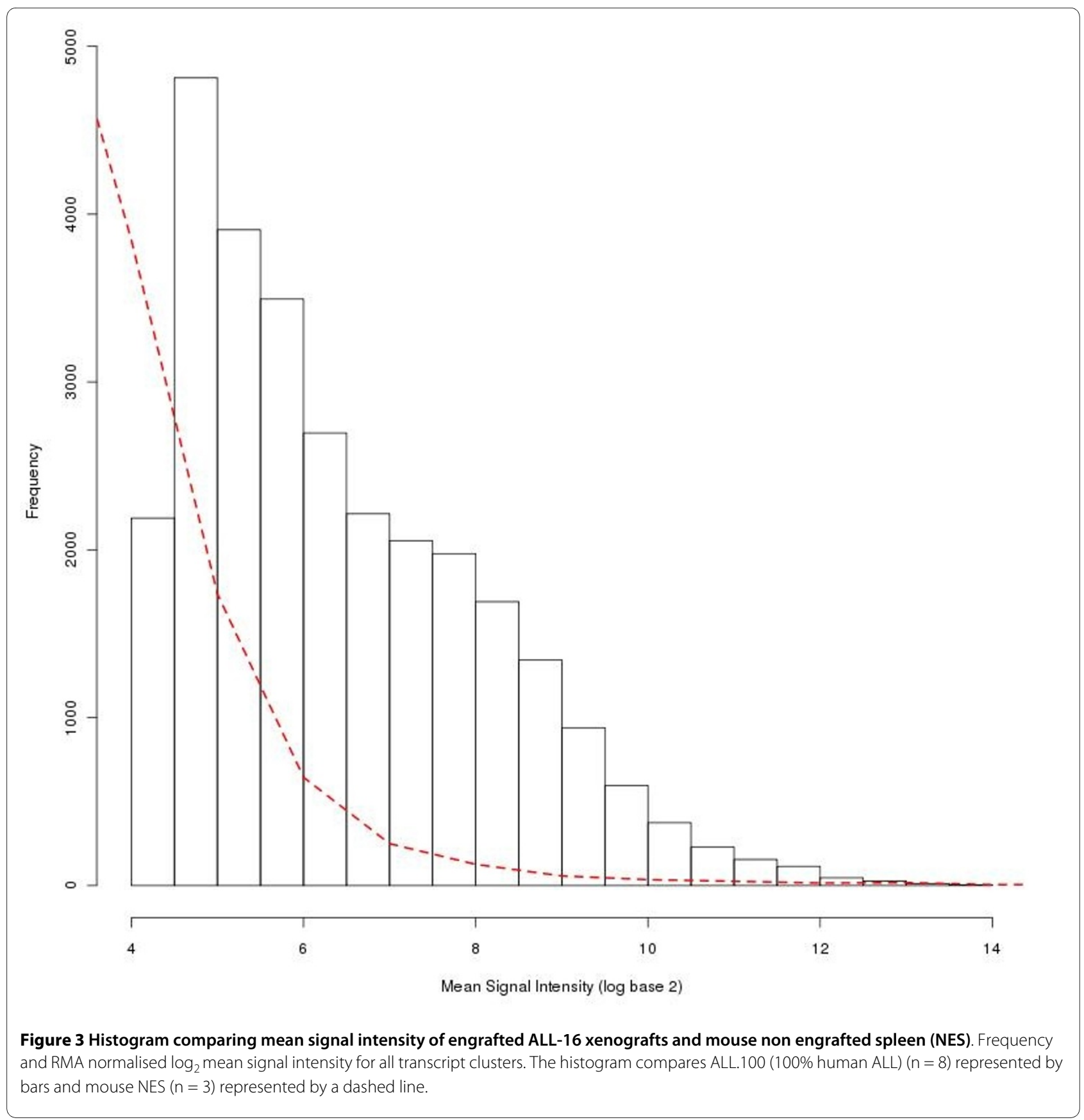

tantly, our findings suggest that spleen is a practical alternative to $\mathrm{BM}$ for profiling xenograft tissue. Microarray analysis revealed no significant difference in the transcriptional signatures of engrafted BM and spleen. Moreover, we determined that the process of leukaemia engraftment is highly concordant and reproducible between independent mice.

\section{Conclusions}

Characterisation of reliable pre-clinical models to identify the most promising new agents to enter clinical trials and molecular events underlying drug resistance is of critical importance. For childhood cancers, xenograft models have been particularly accurate in identifying clinically active agents and effective drug combinations. The continuous NOD/SCID xenograft model for ALL provides a powerful platform with which to study and monitor drug resistance phenotypes in vivo. In this report we have evaluated the accuracy of molecular signatures derived from xenografts. We demonstrate that speciesspecific transcriptional profiles can be obtained from xenografts when high levels of engraftment are achieved 
A

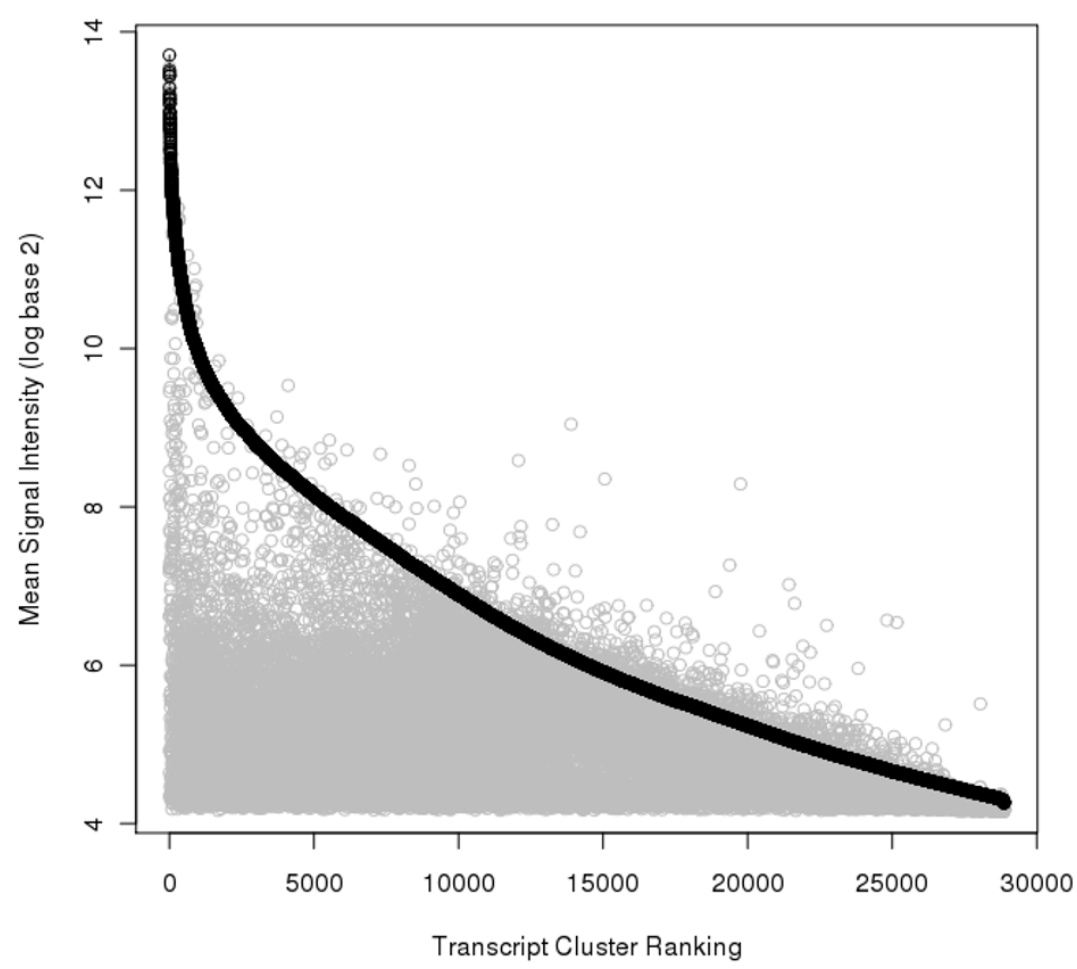

B

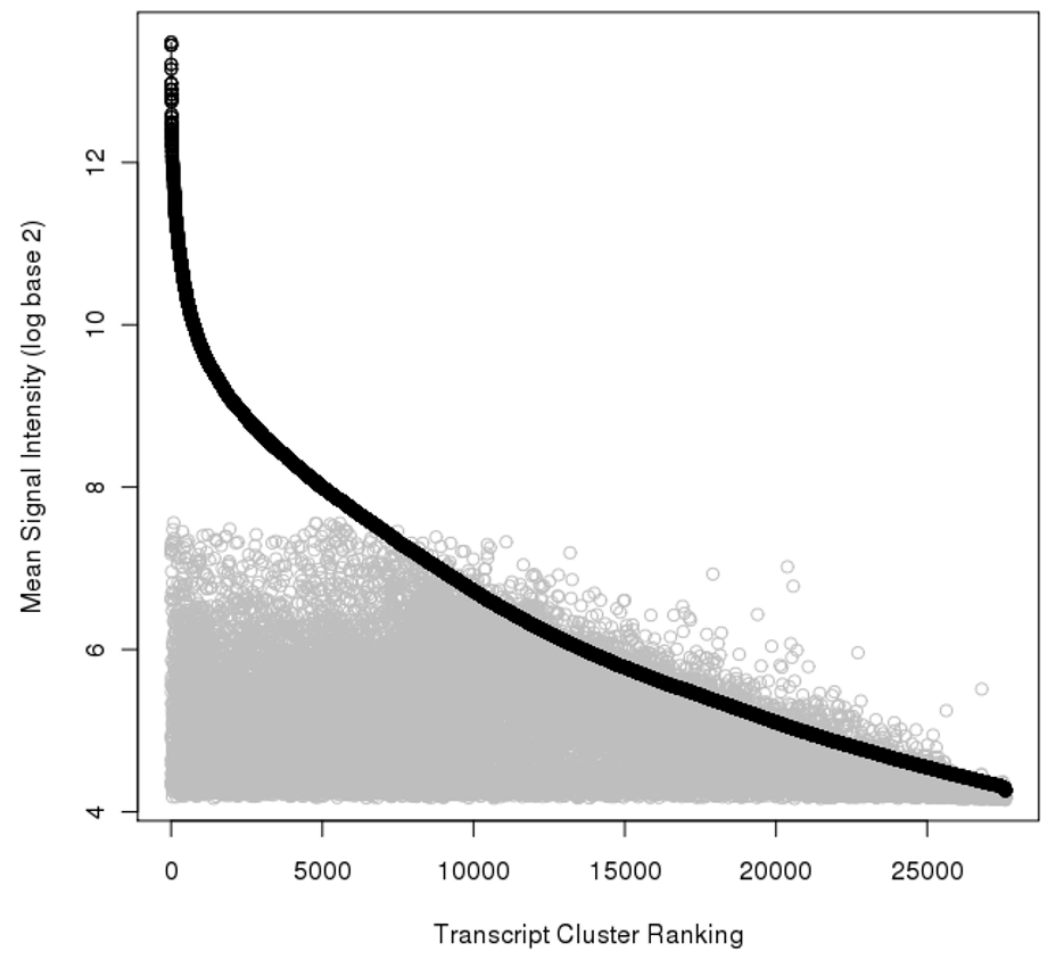

Figure 4 Comparison of ALL.100 ranked transcript cluster signal intensity to mouse non engrafted spleen (NES). Comparison of ranked ALL.100 (100\% human ALL) $(n=8)$, shown in black, and corresponding mouse NES $(n=3)$, shown in grey, mean signal intensity. Transcript clusters are ranked according to descending mean signal intensity of ALL.100. A Without application of filter $\mathbf{B}$ Filtered, via the removal of mouse NES transcript clusters from the top 1\% of normalised expression with a mean signal intensity > 7.56 (log base 2 scale). 
A

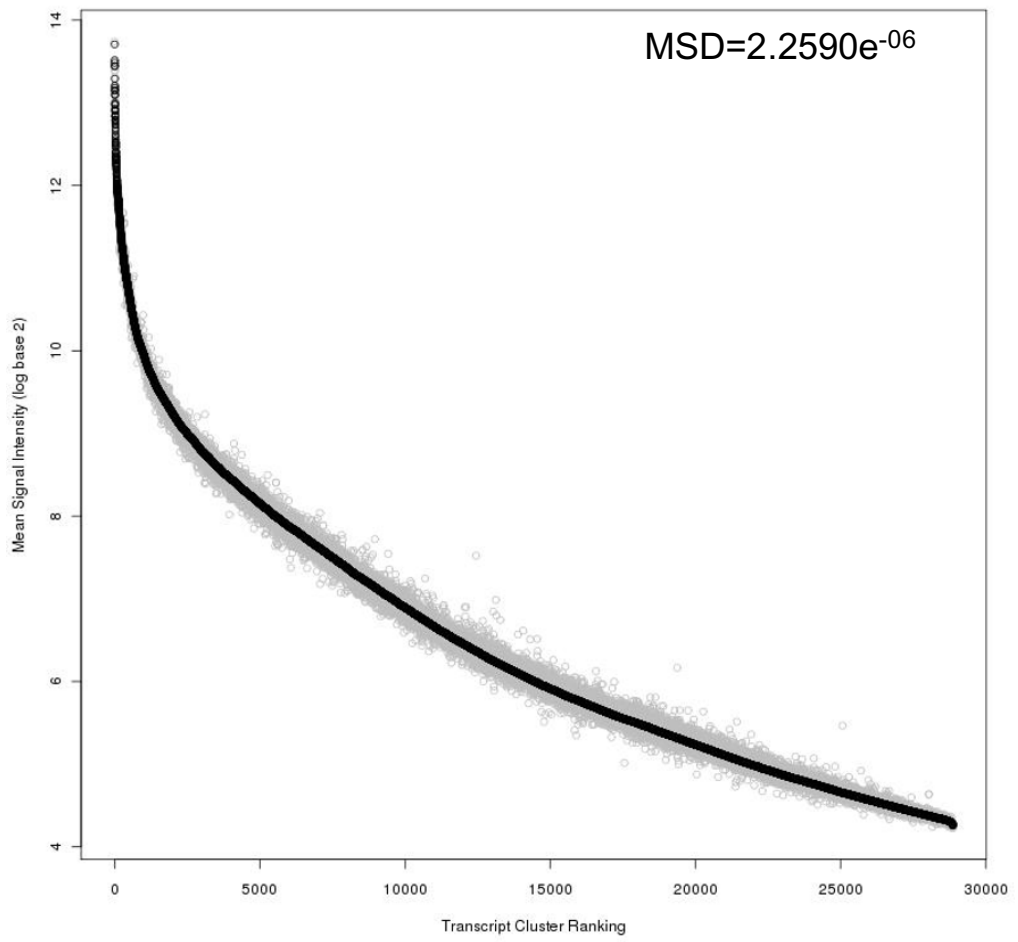

B

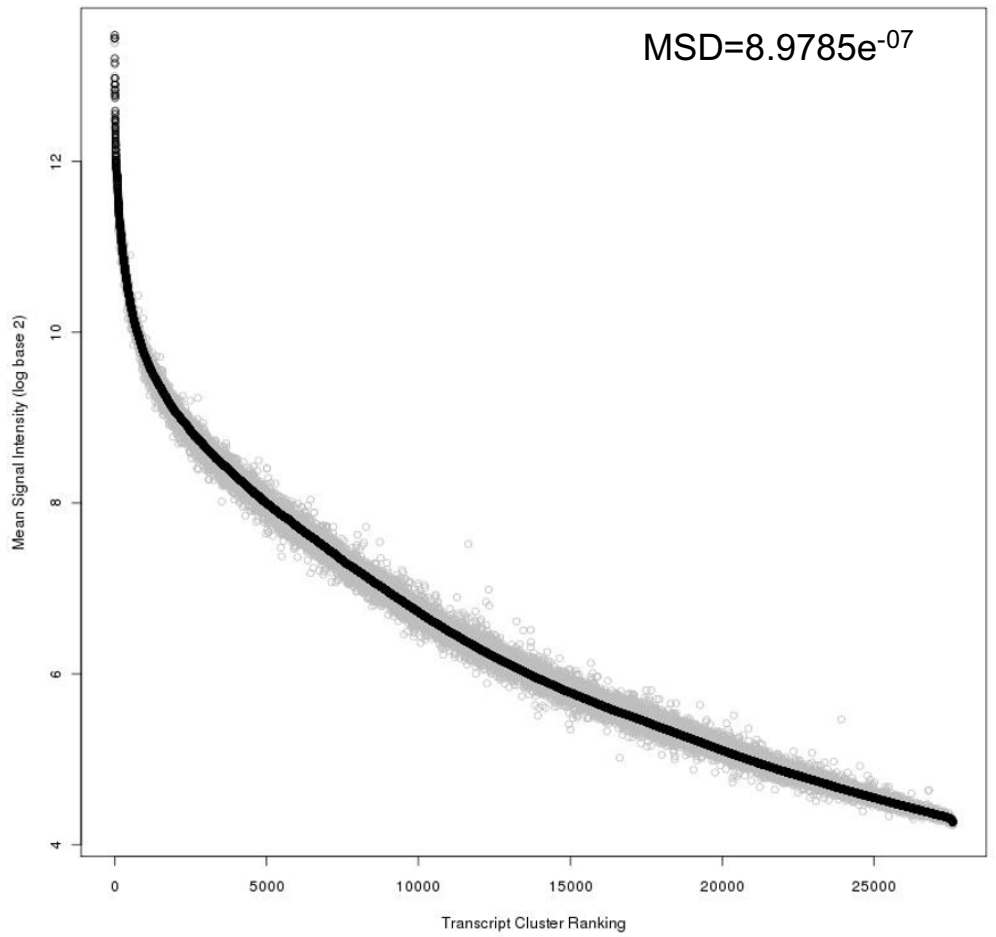

Figure 5 Comparison of ALL.100 ranked transcript cluster signal intensity to ALL.90. Comparison of ranked ALL.100 (100\% human ALL) ( $n=8)$ shown in black and corresponding ALL.90 ( $90 \%$ human) $(n=3)$, shown in grey, mean signal intensity. Transcript clusters are ranked according to descending mean signal intensity of ALL.100. A Without experimental mask B With application of experimental mask. Mean square distance (MSD) was measured between each ALL.100 transcript cluster and the corresponding ALL.90 transcript cluster. 
A

MvA plot ALL.90 versus ALL.100 - Experimental mask approach

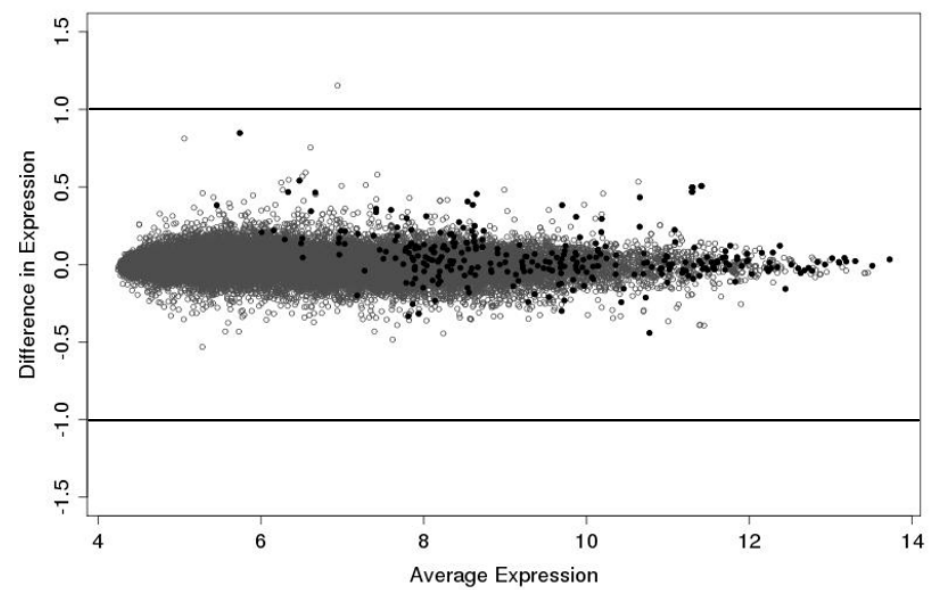

B

MvA plot ALL.90 versus ALL.100 - In silico mask approach

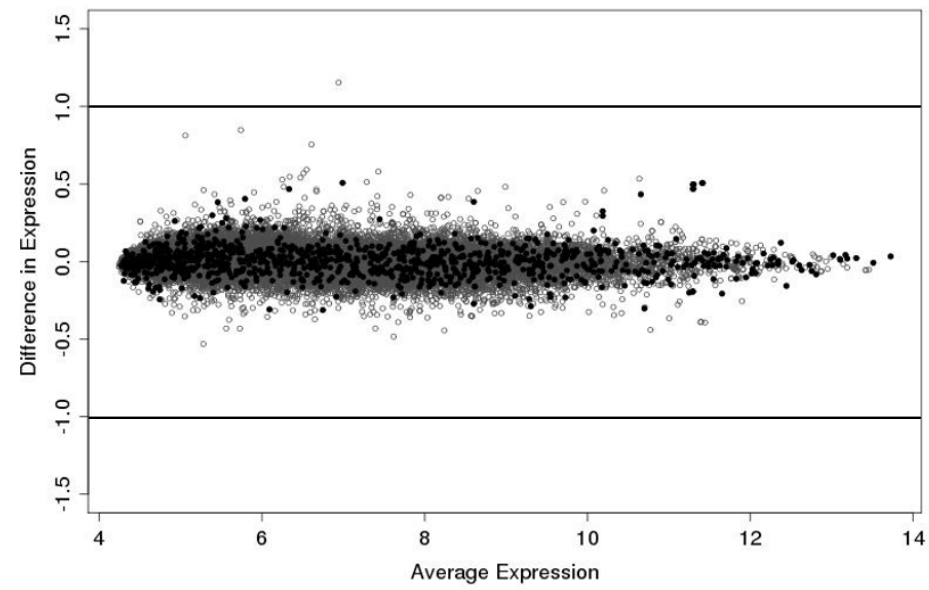

C

MvA plot ALL.90 versus ALL.100- Combined experimental and in silico mask approach

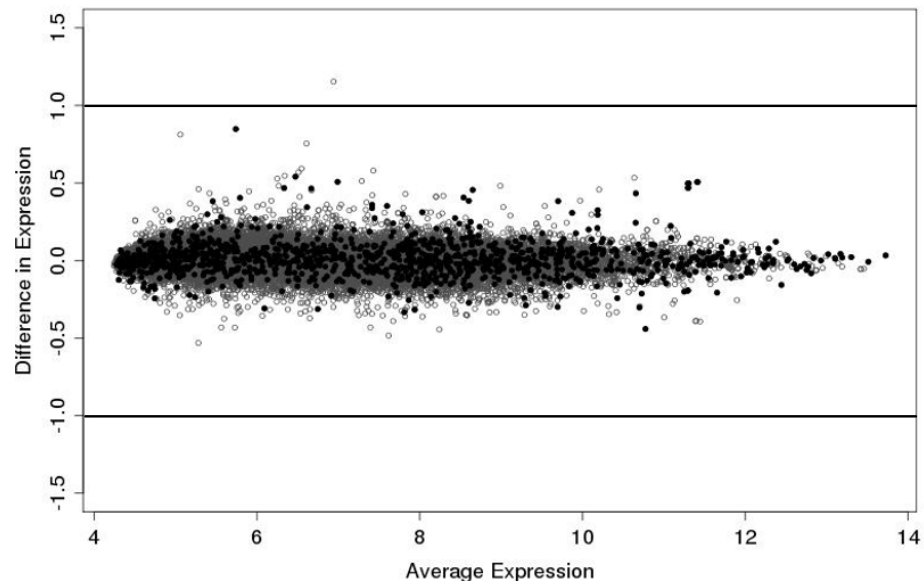

Figure 6 Application of in silico and experimental masks. MvA plots comparing expression differences with expression averages for all transcript clusters. Expression signals from ALL-16 xenografts ALL.100 (100\% human) $(n=8)$ versus ALL.90 ( $90 \%$ human) $(n=3)$ were compared following the application of the $\mathbf{A}$ experimental $\mathbf{B}$ in silico or $\mathbf{C}$ combined masking approaches. Transcript clusters identified as susceptible to cross-species hybridisation are shown in black (.) all others are grey (.). Plots were generated using RMA normalised $\log _{2}$ signal intensities, lines represent 2-fold difference $\left(\log _{2}=1\right)$. 
or with the application of transcript cluster masks. Ultimately, using the continuous xenograft model, this experimental approach will allow the molecular analysis of the effects of individual drugs and drug combinations as well as the identification of biological mechanisms associated with drug resistance phenotypes in vivo.

\section{Methods}

\section{T-ALL Xenograft mouse model}

The University of New South Wales Animal Care and Ethics Committee approved all experimental procedures involving NOD/SCID mice. The ALL-16 xenograft line was established from a patient T-ALL diagnosis sample in the Non-Obese Diabetic/Severe Combined Immunodeficient (NOD/SCID) mouse line as previously reported [2]. Briefly, cells harvested from the spleens of engrafted animals were transplanted into secondary, tertiary and quaternary recipient mice to establish a continuous xenograft model. Quaternary xenografts were used in this study. Engraftment and disease progression was monitored by flow cytometric enumeration of the proportion of human versus mouse CD45+ (\%huCD45+) cells in peripheral blood using established procedures $[2,10]$. When the $\%$ huCD $45+$ reached 50\%, animals were culled and tissue was harvested.

\section{Sample Preparation}

Tissue harvested was femoral bone marrow and spleen from xenografted animals, and spleen from nonengrafted mice. Mononuclear cells from spleen samples were purified by syringe homogenisation, followed by filtration and density gradient centrifugation by FicollPaque $(\mathrm{n}=4)$ (LymphoPrep, Nycomed). Bone marrow cells were collected by flushing both femurs with RPMI 1640 medium (Life Technologies) into a collection tube without density gradient centrifugation $(n=4)$. All cells were cryopreserved for future use in FCS containing 10\% DMSO.

Recovery of human tissue from the engrafted mouse introduces the possibility of contamination from mouse cells. To investigate this further, $95 \%$ and $90 \%$ cell mixtures were prepared using T-ALL from engrafted femoral bone marrow with the non-engrafted mouse spleen from the NOD/SCID mouse strain $(n=3)$.

\section{RNA Isolation}

Total RNA was extracted using TRIZOL reagent (Invitrogen) followed by purification with the RNeasy Mini kit (Qiagen). Samples were ethanol precipitated and quantitated by Nanodrop ND-1000 Spectrophotometry (Thermo Scientific). RNA integrity was assessed using the Agilent 2100 Bioanalyzer and the RNA Nano 6000 kit (Agilent Technologies).

\section{Labelling and Hybridisation}

Samples were labelled according to manufacturers instructions for the GeneChip WT Sense Target Labelling Protocol (Affymetrix). Briefly, 300 ng of total RNA was reverse transcribed into double stranded cDNA using T7- $(\mathrm{N})_{6}$ random primers. An in vitro transcription step was carried out overnight to generate cRNA. Using the cRNA as a template we generated cDNA, which was then fragmented, labelled and hybridised onto Affymetrix Genechip Human Gene 1.0 ST Arrays. The arrays interrogate 28869 well-annotated genes with 764885 probes. The design of the Human Gene 1.0 ST Array was based on the UCSC hg18 and NCBI Build 36 genome sequence assembly. Arrays were washed and stained using the GeneChip Fluidics Station 450 and scanned with the GeneChip Scanner 3000 7G (Affymetrix). All arrays passed quality control criteria as assessed by Expression Console software (Affymetrix).

\section{Data Analysis}

Raw data CEL files were normalised using the Robust Multi-Array (RMA-16) algorithm in GeneSpring version GX 10.0.2 (Agilent Technologies). A mean adjustment of the normalised signal intensity was carried out to account for minor batch effects seen in the data. Analysis was performed using $\mathrm{R}$ version 2.6.2 [29,30]. Microarray data from this study can accessed from ArrayExpress, accession number E-MEXP-2648 http://www.ebi.ac.uk/ arrayexpress

To enable statistically significant data to be interpolated from small sample sizes we used the limma package from Bioconductor [27]. The data was fitted to a linear model and the probability of differential expression was estimated using an empirical Bayes moderated $\mathrm{t}$-test. The $\mathrm{p}$ values were adjusted for multiple testing using a Benjamini-Hochberg correction [28]. Unsupervised hierarchical clustering and Pearson Correlations were generated using the $\log _{2}$ signal intensities following normalisation. MvA plots were generated in $\mathrm{R}$ using the average over the replicates in each group. Mean squared distance (MSD) were calculated in $R$, ranked transcript cluster signal intensity plots and mean signal histograms were also generated in R. Principal Components Analysis (PCA) based on samples and four components was generated in GeneSpring.

\section{Development of a cross-species making approach}

To filter cross-species hybridisation we identified all oligonucleotide probes on the Human GeneChip 1.0 ST array susceptible to spurious signal from mouse RNA using a coupled bioinformatics, in silico and experimental masking approach which is detailed below. 


\section{Cross-species hybridisation derived in silico}

A murine genomic sequence database was compiled from release 54 of the Ensembl Database [31]. All HuGene array annotation files were obtained directly from the Affymetrix website [32]. The probes, probesets and transcript clusters from these files annotated as targeting core human transcripts (i.e. non Affymetrix control probes) were combined, based on their annotated genomic coordinates, to create a mapping table with a three tier hierarchy where probes belong to probesets that in turn belong to transcript clusters (mapping table available on request). The nucleotide sequences for each probe on the HuGene array were obtained from the Affymetrix probe annotation file. The alignment tool BLAT was used to map human probe sequences to the murine genome sequence database. Probes were considered a hit if they matched a database sequence with $100 \%$ identity or with a single base mismatch. The BLAT parameters were optimised to detect the 25 mer probes [33] with tileSize and stepSize set to 12 and 7 respectively. An in house designed Perl script was then used in conjunction with our mapping table to parse the BLAT output files in order to filter out those transcript clusters containing probesets with large numbers of hit probes. Transcript clusters with high numbers of associated hit probes would be deemed candidates for masking. In this filtering process, probes were systematically removed from any probesets with which they were associated. Following this, those probesets with $<50 \%$ of their associated probes remaining were systematically removed from any transcript clusters with which they were associated. Once $\geq 50 \%$ of probesets in a transcript cluster had been removed the transcript cluster was then selected as a candidate for masking.

\section{Cross-species hybridisation derived experimentally}

An experimental approach was employed to examine cross-hybridisation of mouse mRNA to the Human gene array in the absence of human RNA. Three independent non-engrafted mouse spleen (NES) samples were hybridised to the Human Gene 1.0 ST array, CEL files were imported into GeneSpring version GX 10.0.2 and normalised using the Robust Multi-Array (RMA-16) algorithm. The mean signal intensity of the three biological replicates was used to identify cross-hybridising transcript clusters.

\section{Additional material}

Additional file 1 Pearson correlation coefficient matrix. This file contains the Pearson correlation coefficient matrix for transcript cluster expression levels between all transcript clusters for engrafted ALL-16 xenografts and mixing of ALL-16 xenografts with mouse cells (NES).
Additional file 2 Transcript clusters identified as susceptible to crossspecies hybridisation by experimental and in silico masking approaches. This file contains the transcript cluster identity, gene title, gene symbol, chromosomal location and method used for the detection of the 1280 transcript clusters identified as susceptible to cross-species hybridisation.

\section{Authors' contributions}

ALS wrote the manuscript, collected, analysed and summarised the data and principally managed the project. VKP extracted RNA, labelled, hybridised and scanned all microarrays, performed analysis using Genespring and $\mathrm{R}$ and generated the experimental masking approach. RAP maintained mouse lines, dissected tissue and prepared tissue for RNA extraction. MJF participated in the design of the statistical approach and performed statistical analysis using $R$. RWF designed and generated the in silico masking approach. ALS, AHB, URK and RBL made substantial contribution to the conception and design of the experiments. AHB, RBL and URK supported the research and provided significant intellectual contributions to the project and manuscript. All authors read and approved the manuscript.

\section{Acknowledgements}

We gratefully acknowledge the staff and patients of the Centre for Children's Cancer and Blood Disorders, Sydney Children's Hospital, for provision of primary ALL samples from which the continuous xenografts were originally established. This work was supported by the Children's Leukaemia and Cancer Research Foundation (CLCRF), Children's Cancer Institute Australia for Medical Research (CCIA), a fellowship (R.B.L.) and grant from The Australian National Health and Medical Research Council. Children's Cancer Institute Australia for Medical Research is affiliated with The University of New South Wales and Sydney Children's Hospital.

\section{Author Details}

'Division of Children's Leukaemia and Cancer Research, Telethon Institute for Child Health Research, Perth, Western Australia, ${ }^{2}$ Centre for Child Health Research, The University of Western Australia, Perth, Western Australia, ¿Leukaemia Biology, Children's Cancer Institute Australia for Medical Research, Randwick, New South Wales, Australia and 4 Division of Bioinformatics and Biostatistics, Telethon Institute for Child Health Research, Perth, Western Australia

Received: 29 December 2009 Accepted: 21 April 2010

Published: 21 April 2010

\section{References}

1. Macor P, Secco E, Zorzet S, Tripodo C, Celeghini C, Tedesco F: An Update on the Xenograft and Mouse Models Suitable for Investigating New Therapeutic Compounds for the Treatment of B-Cell Malignancies. Current Pharmaceutical Design 2008, 14(21):2023-2039.

2. Lock RB, Liem N, Farnsworth ML, Milross CG, Xue C, Tajbakhsh M, Haber M, Norris MD, Marshall GM, Rice AM: The nonobese diabetic/severe combined immunodeficient (NOD/SCID) mouse model of childhood acute lymphoblastic leukemia reveals intrinsic differences in biologic characteristics at diagnosis and relapse. Blood 2002, 99(11):4100-4108.

3. Kamel-Reid S, Letarte M, Sirard C, Doedens M, Grunberger T, Fulop G, Freedman MH, Phillips RA, Dick JE: A model of human acute lymphoblastic leukemia in immune-deficient SCID mice. Science 1989, 246(4937):1597-1600.

4. Nijmeijer BA, Mollevanger P, van Zelderen-Bhola SL, Kluin-Nelemans HC, Willemze R, Falkenburg JH: Monitoring of engraftment and progression of acute lymphoblastic leukemia in individual NOD/SCID mice. Exp Hematol 2001, 29(3):322-329.

5. Pui CH, Robison LL, Look AT: Acute lymphoblastic leukaemia. Lancet 2008, 371(9617):1030-1043.

6. Kamb A: What's wrong with our cancer models? Nat Rev Drug Discov 2005, 4(2):161-165.

7. Baersch G, Mollers T, Hotte A, Dockhorn-Dworniczak B, Rube C, Ritter J, Jurgens H, Vormoor J: Good engraftment of B-cell precursor ALL in NOD-SCID mice. Klin Padiatr 1997, 209(4):178-185.

8. Borgmann A, Baldy C, von Stackelberg A, Beyermann B, Fichtner I, Nurnberg $P$, Henze G: Childhood all blasts retain phenotypic and 
genotypic characteristics upon long-term serial passage in NOD/SCID mice. Pediatr Hematol Oncol 2000, 17(8):635-650.

9. Dialynas DP, Shao L, Billman GF, Yu J: Engraftment of human T-cell acute lymphoblastic leukemia in immunodeficient NOD/SCID mice which have been preconditioned by injection of human cord blood. Stem Cells 2001, 19(5):443-452.

10. Liem NL, Papa RA, Milross CG, Schmid MA, Tajbakhsh M, Choi S, Ramirez $C D$, Rice AM, Haber M, Norris MD, et al: Characterization of childhood acute lymphoblastic leukemia xenograft models for the preclinical evaluation of new therapies. Blood 2004, 103(10):3905-3914.

11. Houghton PJ, Morton CL, Tucker C, Payne D, Favours E, Cole C, Gorlick R, Kolb EA, Zhang W, Lock R, et al: The pediatric preclinical testing program: description of models and early testing results. Pediatr Blood Cancer 2007, 49(7):928-940.

12. Neale G, Su X, Morton CL, Phelps D, Gorlick R, Lock RB, Reynolds CP, Maris $J M$, Friedman HS, Dome J, et al:: Molecular characterization of the pediatric preclinical testing panel. Clin Cancer Res 2008, 14(14):4572-4583

13. Peterson JK, Houghton PJ: Integrating pharmacology and in vivo cancer models in preclinical and clinical drug development. Eur J Cancer 2004, 40(6):837-844

14. Whiteford CC, Bilke S, Greer BT, Chen Q, Braunschweig TA, Cenacchi N, Wei JS, Smith MA, Houghton P, Morton C, et al: Credentialing preclinical pediatric xenograft models using gene expression and tissue microarray analysis. Cancer Res 2007, 67(1):32-40.

15. Beesley AH, Cummings AJ, Freitas JR, Hoffmann K, Firth MJ, Ford J, de Klerk $\mathrm{NH}$, Kees UR: The gene expression signature of relapse in paediatric acute lymphoblastic leukaemia: implications for mechanisms of therapy failure. Br J Haematol 2005, 131(4):447-456.

16. Hoffmann K, Firth MJ, Beesley AH, de Klerk NH, Kees UR: Translating microarray data for diagnostic testing in childhood leukaemia. $B M C$ Cancer 2006, 6:229.

17. Gottardo NG, Hoffmann K, Beesley AH, Freitas JR, Firth MJ, Perera KU, de Klerk NH, Baker DL, Kees UR: Identification of novel molecular prognostic markers for paediatric T-cell acute lymphoblastic leukaemia. Br J Haematol 2007, 137(4):319-328.

18. Hoffmann K, Firth MJ, Beesley AH, Freitas JR, Ford J, Senanayake S, de Klerk $\mathrm{NH}$, Baker DL, Kees UR: Prediction of relapse in paediatric pre-B acute lymphoblastic leukaemia using a three-gene risk index. Br J Haematol 2008, 140(6):656-664.

19. Beesley AH, Firth MJ, Ford J, Weller RE, Freitas JR, Perera KU, Kees UR: Glucocorticoid resistance in T-lineage acute lymphoblastic leukaemia is associated with a proliferative metabolism. Br J Cancer 2009, 100(12):1926-1936

20. Boag JM, Beesley AH, Firth MJ, Freitas JR, Ford J, Hoffmann K, Cummings AJ, de Klerk NH, Kees UR: Altered glucose metabolism in childhood preB acute lymphoblastic leukaemia. Leukemia 2006, 20(10):1731-1737.

21. Boag JM, Beesley AH, Firth MJ, Freitas JR, Ford J, Brigstock DR, de Klerk NH, Kees UR: High expression of connective tissue growth factor in pre-B acute lymphoblastic leukaemia. Br J Haematol 2007, 138(6):740-748.

22. Creighton C, Kuick R, Misek DE, Rickman DS, Brichory FM, Rouillard JM, Omenn GS, Hanash S: Profiling of pathway-specific changes in gene expression following growth of human cancer cell lines transplanted into mice. Genome Biol 2003, 4(7):R46.

23. Creighton CJ, Bromberg-White JL, Misek DE, Monsma DJ, Brichory F, Kuick R, Giordano TJ, Gao W, Omenn GS, Webb CP, et al:: Analysis of tumor-host interactions by gene expression profiling of lung adenocarcinoma xenografts identifies genes involved in tumor formation. Mol Cancer Res 2005, 3(3):119-129.

24. Harrell JC, Dye WW, Harvell DM, Sartorius CA, Horwitz KB: Contaminating cells alter gene signatures in whole organ versus laser capture microdissected tumors: a comparison of experimental breast cancers and their lymph node metastases. Clin Exp Metastasis 2008, 25(1):81-88.

25. Naef F, Huelsken J: Cell-type-specific transcriptomics in chimeric models using transcriptome-based masks. Nucleic Acids Res 2005, 33(13): 1111.

26. Wilson CL, Sims AH, Howell A, Miller CJ, Clarke RB: Effects of oestrogen on gene expression in epithelium and stroma of normal human breast tissue. Endocr Relat Cancer 2006, 13(2):617-628.

27. Smyth GK: Linear Models and Empirical Bayes Methods for Assessing Differential Expression in Microarray Experiments. Statistical Applications in Genetics and Molecular Biology 2004, 3(1):
28. Benjamini Y, Hochberg Y: Controlling the False Discovery Rate: A Practical and Powerful Approach to Multiple Testing. Journal of the Royal Statistical Society Series B (Methodological) 1995, 57(1):289-300.

29. Ihaka R, Gentleman R: R: A Language for Data Analysis and Graphics. Journal of Computational and Graphical Statistics 1996, 5(3):299-314.

30. Team RDC: R: A language and environment for statistical computing. $R$ Foundation for Statistical Computing. Vienna, Austria; 2008.

31. Ensembl website [http://www.ensembl.org/index.html]

32. Affymetrix website [http://www.affymetrix.com/index.affx]

33. Blat parameters [http://genome.ucsc.edu/FAQ/FAQblat\#blat8]

doi: 10.1186/1471-2164-11-256

Cite this article as: Samuels et al., Validation of a mouse xenograft mode system for gene expression analysis of human acute lymphoblastic leukaemia BMC Genomics 2010, 11:256

\section{Submit your next manuscript to BioMed Central and take full advantage of:}

- Convenient online submission

- Thorough peer review

- No space constraints or color figure charges

- Immediate publication on acceptance

- Inclusion in PubMed, CAS, Scopus and Google Scholar

- Research which is freely available for redistribution
C Biomed Central 